\title{
Flexibilidad y teletrabajo: un estudio bibliométrico sobre la producción científica
}

Jackson André da Silva

\begin{abstract}
Doutorando pela Universidad de Zaragoza Mestre em Administração pela ESAG/UDESC Analista Técnico do Sebrae/SC - Serviço de Apoio às Micro e Pequenas Empresas de Santa Catarina
\end{abstract}

http://dx.doi.org/10.1590/1981-5344/1884

Este artículo tiene como objetivo estudiar y analizar un conjunto de autores, periódicos y palabras clave que se relacionan a flexibilidad y teletrabajo. Para ello, se aplicó la metodología de revisión sistemática de la literatura en cuatro bases de datos científicas, proceso que identificó 80 estudios sobre el tema buscado. Los resultados muestran que las investigaciones más relevantes, de acuerdo con el número de citaciones, son de países anglosajones (EE.UU., Canadá y Reino Unido, respectivamente), que hay poca investigación en colaboración internacional y que los trabajos más citados tienen como enfoque las características de los teletrabajadores y el impacto del teletrabajo en la conciliación personal y profesional.

Palabras clave: Flexibilidad; Teletrabajo; Levantamiento bibliográfico.

\section{Flexibility and telework: a study about scientific production analysis.}

This article aims to collecting and analyzing a set of authors, journals and keywords that relate issues flexibility and telework. For this, we applied the methodology of systematic literature review in four scientific databases, process that identified 80 studies on the topic searched. The results show that work and research more relevant, according to the number of citations, are from anglo saxon countries (United States, Canada and England, respectively); that there is little research in international partnership and that the most 
cited papers have focus on the characteristics of teleworkers and the impact of telework in reconciling personal and professional life.

Keywords: Flexibility; Telework; Systematic literature review.

Recebido em 03.10.2013 Aceito em 07.12.2013

\section{Introducción}

Las incertidumbres del entorno empresarial llevan a las empresas a adoptar prácticas más flexibles, sean ellas estratégicas u operativas, siendo así capaces de responder rápidamente a las nuevas demandas. El tema flexibilidad ha sido investigado desde los años 30, pero los estudios han ganado impulso en los años 80 y 90 .

Una forma de flexibilizar la empresa es la adopción del teletrabajo, que se centra en la gestión de las actividades y no se limita al sitio de la empresa (DAVENPORT; PEARLSON, 1998). Por lo tanto, es posible ofrecer beneficios tales como la reducción de los viajes y el horario de trabajo flexible, facilitando la conciliación de la vida laboral y personal (de LUIS et al., 2006).

Aunque hay cada vez más estudios publicados con interés en el tema, como se detallará en este artículo, todavía es necesario un estudio bibliométrico, siguiendo criterios científicos para transformar los datos cuantitativos en información para los investigadores de este campo de conocimiento.

Por lo tanto, este estudio se justifica por la importancia de la identificación de los principales autores y trabajos sobre flexibilidad y teletrabajo, así como por identificar las deficiencias que pueden ayudar a los investigadores que están interesados en iniciar una investigación sobre este tema y avanzar en el desarrollo de la ciencia.

Así, este trabajo tiene como objetivo seleccionar y analizar un conjunto de autores, periódicos y palabras clave que se relacionan con los temas flexibilidad y teletrabajo. Para lograr el objetivo, se realizó un mapeo de las bases de datos mediante los términos de búsqueda "flexibilidad y teletrabajo" con el fin de contribuir a una mejor comprensión de las características de los estudios sobre el tema, tratando de identificar las relaciones y tendencias en la intersección de las dos áreas de investigación.

En la segunda sección se presentan los conceptos de flexibilidad y teletrabajo. En la sección tercera la metodología aplicada en este estudio, seguida por la presentación y análisis de los resultados. Por último, en la quinta sección, se presentan las conclusiones y recomendaciones para trabajos futuros. 


\section{Flexibilidad y teletrabajo}

El estudio de la flexibilidad empezó con el trabajo de Hart (1937) y Stigler (1939) y trataban de comprender cómo las instalaciones industriales podrían producir algo que no estaba previsto. Aunque los primeros estudios encontrados han sido publicados hace mucho tiempo, el interés en el tema ha ganado impulso en los años 80 y 90, con la aparición de diversos conceptos y tipologías.

Sin embargo, todavía no existe una definición única de lo que es la flexibilidad y la forma en que se puede medirla, ya que las definiciones suelen incluir situaciones o problemas específicos de gestión (UPTON, 1994). Estos problemas pueden tener su origen en el intento de adaptar la organización para satisfacer las necesidades del mercado, donde la flexibilidad se presenta como una respuesta estratégica que soportar la incertidumbre y la variabilidad de los productos y procesos de producción.

Según Sánchez (1995) la flexibilidad depende conjuntamente de la flexibilidad de los recursos y de la flexibilidad de coordinación de la empresa en utilizarlos. Una característica importante en este contexto es el recurso humano, ya que actúa como un recurso y, al mismo tiempo, es responsable de por la coordinación.

De acuerdo con Wright y Snell (1998), los empleados tienen las habilidades y comportamientos que ofrecen a la empresa una serie de alternativas, y que se ellas fueren bien gestionadas pueden generar una ventaja competitiva. Aunque las estrategias de recursos humanos no proporcionan una conexión causal directa con el resultado, pueden influir indirectamente en ellos mediante la flexibilidad.

Sánchez y otros (2009) afirman que una de las prácticas adoptadas por las empresas es la posibilidad de los empleados trabajaren desde su casa o de despachos virtuales. Esta práctica también es conocida como teletrabajo. Según los autores, esta opción ofrece a los empleados la posibilidad de reducir el desplazamiento entre su hogar y la empresa, así como la conciliación de las actividades personales y de trabajo.

Para Davenport y Pearlson (1998) el teletrabajo tiene como enfoque la gestión del las actividades, independientemente del lugar donde se llevan a cabo, haciendo posible a través del uso de las Tecnologías de Información y Comunicación (TIC). Su adopción, según de Luis y otros (2006), puede traer resultados positivos para el medio ambiente derivados de los ahorros de combustible asociados a la reducción de los desplazamientos de los empleados. Estos resultados positivos también pueden incidir en la vida personal, con la posibilidad de gestionar mejor el uso del tiempo entre el trabajo, el cuidado familiar y el ocio.

\section{Metodología}

Para la preparación de este artículo se utilizó la metodología de revisión sistemática de la literatura para mapear las producciones científicas que exploran la relación entre flexibilidad y teletrabajo. Este 
tipo de investigación proporciona un resumen de las evidencias relacionadas a la aplicación de criterios científicos de búsqueda establecidos, evaluación crítica y síntesis de la información seleccionada. Las revisiones sistemáticas pueden identificar lagunas que necesitan evidencias en el campo de investigación, ayudando en la orientación para futuros estudios (NATIONAL HEALTH SERVICE - NHS, 2001). Así, de acuerdo con Jackson (2004), la revisión sistemática se compone de siete etapas, a saber: planificar la revisión, formular la pregunta, hacer la búsqueda, seleccionar los datos, evaluar críticamente, sintetizar e interpretar los resultados.

En respuesta a la primera fase propuesta, se procedió a la planificación de la revisión sistemática. Para ella, fueron seleccionadas las bases de datos utilizadas en este estudio, la delimitación del tema, que ha conducido a pregunta de investigación, los tipos de documentos, idiomas, año de publicación y los criterios de inclusión y exclusión de los estudios encontrados. Se eligieron cuatro bases de datos, a saber: SciELO, ScienceDirect, Scopus y Web of Science. La elección de estas bases se realizó debido al facto de que ellas son recomendadas pela Coordenação de Aperfeiçoamento de Pessoal de Nível Superior (CAPES) y por cubrir una gran cantidad de periódicos relevantes sobre el tema buscado.

El segundo paso propuesto por Jackson (2004) para llevar a cabo la revisión sistemática pide la formulación de una pregunta de investigación. Por lo tanto, con base en la definición propuesta en la etapa de planificación, fue configurada la siguiente pregunta: ¿Cuáles son los trabajos científicos que exploran la relación entre flexibilidad $y$ teletrabajo? Basado en esta cuestión, las palabras de búsqueda fueron definidas con la intersección de dos conjuntos de palabras (flexible 0 flexibility $O$ flexibilidad $O$ flexibilidade) y (telework $O$ teletrabajo $O$ teletrabalho). Para aumentar la incidencia de trabajos, fueron consideradas las variaciones de las palabras elegidas con el uso de algunos caracteres especiales como "*" (que sustituye a los números y letras, por lo que se puede utilizar para localizar cualquier número o un carácter que indica que puede o no puede estar presente). Ejemplo: teletraba* busca teletrabajo, teletrabalho u otras variaciones.

Por lo tanto, la etapa de realizar la búsqueda siguió la planificación pre-establecida, siendo realizada entre los días 9 y 11 de junio de 2013, con las siguientes palabras: (flexib*) AND (telework OR teletraba*). Se destaca que las palabras fueron buscadas sólo en el título, en el resumen y en las palabras clave, para aumentar la adhesión a la pregunta de investigación.

Fueron encontrados 137 documentos do tipo Articles al final de la etapa 3 y los otros tipos de documentos fueron descartados. En cuanto al idioma y al año de publicación, no se ha aplicado ningún tipo de filtro, ya que se tenía el objetivo de recuperar la máxima cantidad de trabajos publicados sobre el tema.

Los criterios de selección de artículos involucraron la lectura del título y resumen para asegurar que el contenido respondiera a la pregunta de investigación. Artículos duplicados (encontrados en más de una base 
de datos) fueron considerados solo una vez y los artículos cuyo contenido se ha mostrado incompatible han sido eliminados, siguiendo el flujo de la Figura 1.

Figura 1 - Criterios de selección de los artículos

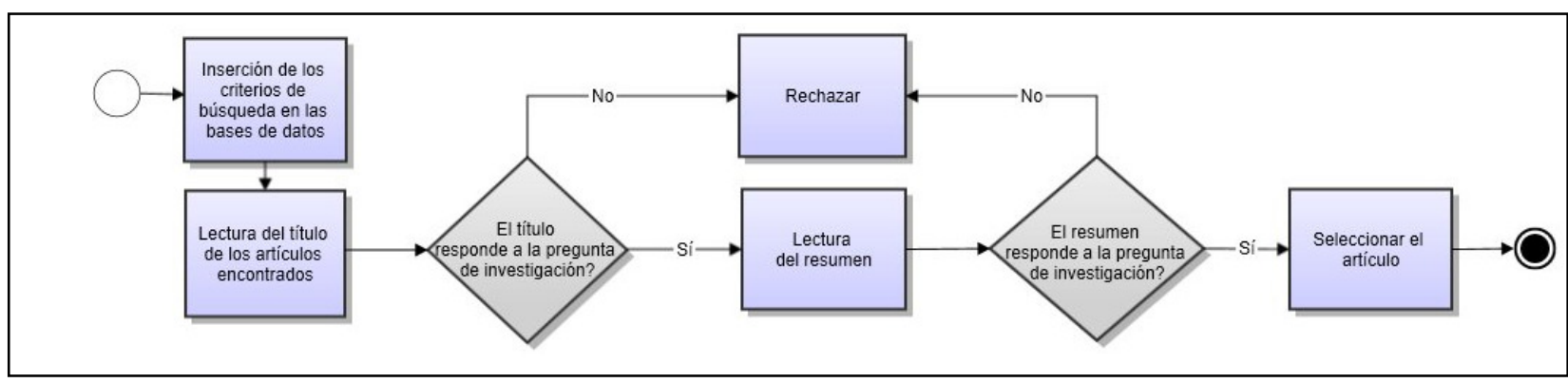

Fuente: Desarrollada por el autor.

Después de leer el título y resumen, nueve artículos fueron excluidos por no exploraren la relación entre flexibilidad y teletrabajo y otros 48 porque estaban repetidos, dejando al final sólo 80 artículos únicos. La Tabla 1 muestra la cantidad de artículos encontrados y seleccionados por base de datos.

Tabla 1 - Artículos seleccionados por base de datos

\begin{tabular}{ccccc}
\hline Base de Datos & Total de artículos & Sin adhesión & Repetidos & Seleccionados \\
\hline \hline SciELO & 02 & 02 & - & - \\
WOS & 62 & 03 & - & 59 \\
Scopus & 61 & 03 & 37 & 21 \\
ScienceDirect & 12 & 01 & 11 & - \\
\hline TOTAL & $\mathbf{1 3 7}$ & $\mathbf{0 9}$ & $\mathbf{4 8}$ & $\mathbf{8 0}$ \\
\hline
\end{tabular}

Fuente: Desarrollada por el autor.

Se sabe que uno de los principales objetivos esperados por los investigadores es que su trabajo sea reconocido, y este reconocimiento se mide basándose en la cantidad de citaciones que recibe un trabajo en particular. Por lo tanto, se cree que para el presente trabajo este dato sea un indicador relevante.

Dada la amplitud de bases de datos consultadas, se utilizó el programa HistCite para relacionar los artículos que se citan entre las publicaciones seleccionadas y este indicador fue llamado de Internal Citation Score (ICS). El sitio de Google Académico también fue utilizado con el objetivo de encontrar la cantidad total de citaciones recibidas por los artículos y este indicador fue llamado Google Scholar Citation Score (GSCS).

Al final de la fase de selección de datos fueron encontrados a 80 artículos científicos únicos y adherentes a la pregunta de investigación. Para trabajar el conjunto de los datos fue realizada la exportación de la información necesaria para archivos de texto (tipo ISI y RIS), que después fueron importados al programa HistCite, que permitió la realización de las etapas de evaluación crítica, síntesis e interpretación de los resultados, que se describirá en la siguiente sección. 


\section{Presentación y análisis de los resultados}

Los resultados generales obtenidos en el análisis bibliográfico (fases de evaluación, síntesis e interpretación de los resultados), están sintetizados en la Tabla 2.

Tabla 2. Resultados generales del análisis bibliográfico

\begin{tabular}{ccc}
\hline Criterio & & Valor \\
\hline & Artículos & 80 \\
& Autores & 152 \\
& Idiomas & 06 \\
& Países & 20 \\
& Palabras clave & 421 \\
Periódicos & 59 \\
Referencias citadas & 3.121 \\
\hline
\end{tabular}

Fuente: Desarrollada por el autor.

El artículo más antiguo de la búsqueda fue publicado en 1984. También fueron encontrados publicaciones en los años de 1986 y 1990, lo que demuestra que el interés por el tema hasta entonces era bajo. Sin embargo, desde 1995 hubo un aumento en el interés por el tema y en todos los años posteriores fueron encontrados al menos un artículo publicado, llegando a nueve publicaciones en 2012. La Figura 2 muestra el registro completo de los artículos de acuerdo con el año en que fueron publicados.

Figura 2 - Cantidad de artículos por ano

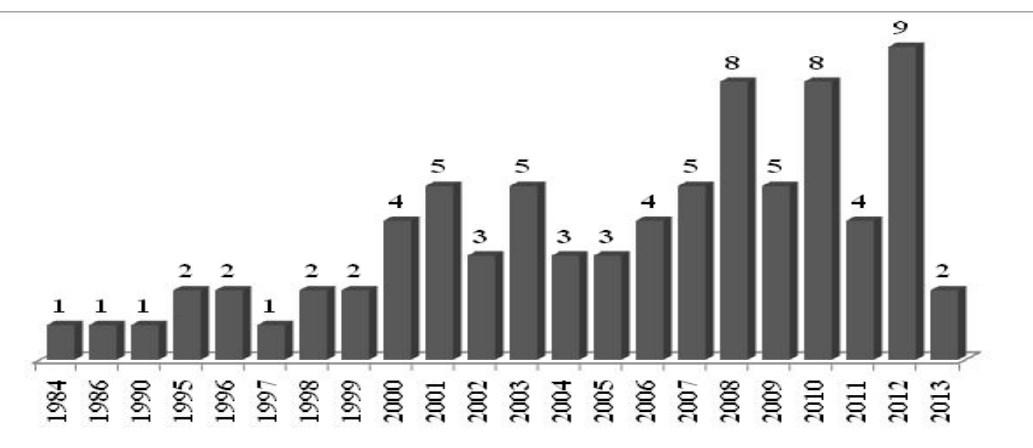

Fuente: Desarrollada por el autor.

En cuanto a los medios de comunicación, fueron encontrados 59 periódicos que publicaron algún artículo sobre el tema buscado. Para identificar los periódicos más relevantes fue utilizada la ley de dispersión creada por Bradford (1934). De acuerdo con Araujo (2006), la ley de dispersión permite estimar un grupo de periódicos de mayor calidad y relevancia para una determinada área de investigación. Entonces, utilizándose de esta ley fue posible identificar los periódicos que publicaron el mayor número de artículos sobre flexibilidad y teletrabajo.

En la Tabla 3 constan descritos los nueve periódicos que componen el núcleo de mayor calidad y relevancia para el tema buscado, el porcentual de artículos publicados en cada uno y sus respectivos índices de citaciones (ICS y GSCS). Los periódicos que componen los otros dos 
grupos de extensión fueron aglutinados y juntos totalizaron $62,5 \%$ de las publicaciones.

Tabla 3 - Periódicos en que los artículos fueron publicados

\begin{tabular}{|c|c|c|c|c|}
\hline \multirow{2}{*}{ Periódicos } & \multirow{2}{*}{$\begin{array}{c}\mathrm{N}^{\circ} \mathrm{de} \\
\text { artículos }\end{array}$} & \multirow{2}{*}{$\%$} & \multicolumn{2}{|c|}{ Citaciones } \\
\hline & & & ICS $^{*}$ & GSCS $^{\star}$ \\
\hline New technology work and employment & 11 & $13,8 \%$ & 38 & 305 \\
\hline Journal of vocational behavior & 04 & $5,0 \%$ & 16 & 420 \\
\hline Gender work and organization & 03 & $3,8 \%$ & 06 & 225 \\
\hline British journal of management & 02 & $2,5 \%$ & 01 & 13 \\
\hline Informacios tarsadalom & 02 & $2,5 \%$ & 00 & 00 \\
\hline International journal of human resource management & 02 & $2,5 \%$ & 00 & 62 \\
\hline Journal of applied psychology & 02 & $2,5 \%$ & 06 & 170 \\
\hline Journal of organizational computing and electronic commerce & 02 & $2,5 \%$ & 01 & 81 \\
\hline Work-a journal of prevention assessment \& rehabilitation & 02 & $2.5 \%$ & 00 & 00 \\
\hline Otros periódicos (50) & 50 & $62,5 \%$ & 44 & 1.292 \\
\hline TOTAL & 80 & $100,0 \%$ & 112 & 2.568 \\
\hline
\end{tabular}

* Internal Citation Score

** Google Scholar Citation Score

Fuente: Desarrollada por el autor.

Aunque el principal medio de comunicación utilizado por los autores ha sido el periódico New Technology Work and Employment con 11 trabajos publicados, el Journal of Vocational Behavior demostró que tiene una mayor visibilidad, ya que presentó un mayor GSCS, incluso con sólo cuatro artículos publicados.

Ninguna restricción fue asignada al país de origen de las publicaciones, ya que la intención era encontrar la procedencia de las investigaciones en este tema. El país que presentó el mayor número de publicaciones fue EE.UU. con 26 artículos, seguido por Reino Unido con 13 y Canadá con 10 (Tabla 4).

Tabla 4 - País de origen de las publicaciones

\begin{tabular}{|c|c|c|c|c|}
\hline \multirow{2}{*}{ País } & \multirow{2}{*}{$\begin{array}{c}\mathbf{N}^{\circ} \mathrm{de} \\
\text { artículos }\end{array}$} & \multirow{2}{*}{$\%$} & \multicolumn{2}{|c|}{ Citaciones } \\
\hline & & & ICS & GSCS \\
\hline EE.UU. & 26 & $31 \%$ & 45 & 1.529 \\
\hline Reino Unido & 13 & $15 \%$ & 42 & 602 \\
\hline Canadá & 10 & $12 \%$ & 24 & 403 \\
\hline Australia & 05 & $06 \%$ & 01 & 05 \\
\hline Alemania & 05 & $06 \%$ & 01 & 105 \\
\hline España & 05 & $06 \%$ & 00 & 83 \\
\hline Países Bajos & 03 & $04 \%$ & 00 & 04 \\
\hline Bélgica & 02 & $02 \%$ & 01 & 28 \\
\hline Brasil & 02 & $02 \%$ & 01 & 02 \\
\hline Chipre & 02 & $02 \%$ & 01 & 13 \\
\hline Francia & 02 & $02 \%$ & 00 & 05 \\
\hline Hungría & 02 & $02 \%$ & 00 & 00 \\
\hline Otros países (08) & 08 & $09 \%$ & 04 & 199 \\
\hline TOTAL & 85 & $100 \%$ & 120 & 2.978 \\
\hline
\end{tabular}

Fuente: Desarrollada por el autor.

Cinco investigaciones fueron conducidas en co-autoría entre países, lo que demuestra poca colaboración internacional entre investigadores que trabajan en este tema. De estos cinco estudios, cuatro fueron hechos en asociación con EE.UU. (Alemania, Canadá, Dinamarca y Japón) y el quinto se llevó a cabo entre investigadores de los Países Bajos y Nueva Zelandia. 
Como los tres países con el mayor número de publicaciones son anglosajones y juntos representan el $58 \%$ del total publicado sobre el tema, el idioma inglés fue lo que prevaleció en los artículos seleccionados (85\%). Sin embargo, también hubo otros idiomas como alemán, francés, español, portugués y húngaro (Tabla 5 ).

Tabla 5 - Idiomas de los artículos

\begin{tabular}{ccccc}
\hline \multirow{2}{*}{ Idioma } & $\begin{array}{c}\mathbf{N}^{\circ} \text { de } \\
\text { artículos }\end{array}$ & $\%$ & \multicolumn{2}{c}{ Citaciones } \\
\cline { 4 - 5 } & 69 & $85 \%$ & 112 & 2.551 \\
\hline \hline Inglés & 03 & $04 \%$ & 00 & 10 \\
Alemán & 03 & $04 \%$ & 00 & 05 \\
Francés & 02 & $03 \%$ & 00 & 02 \\
Español & 02 & $03 \%$ & 00 & 00 \\
Húngaro & 01 & $01 \%$ & 00 & 00 \\
Portugués & $\mathbf{8 0}$ & $\mathbf{1 0 0 \%}$ & $\mathbf{1 1 2}$ & $\mathbf{2 . 5 6 8}$ \\
\hline TOTAL & $\mathbf{8 0}$
\end{tabular}

Fuente: Desarrollada por el autor.

Para ayudar en el análisis de los términos más inseridos como palabras clave, fue preparada la Figura 3 , que es una nube de tags que enseña el tamaño de las palabras en función del número de veces en que fueron mencionadas.

Figura 3 - Nube de tags con las principales palabras clave inseridas en los artículos

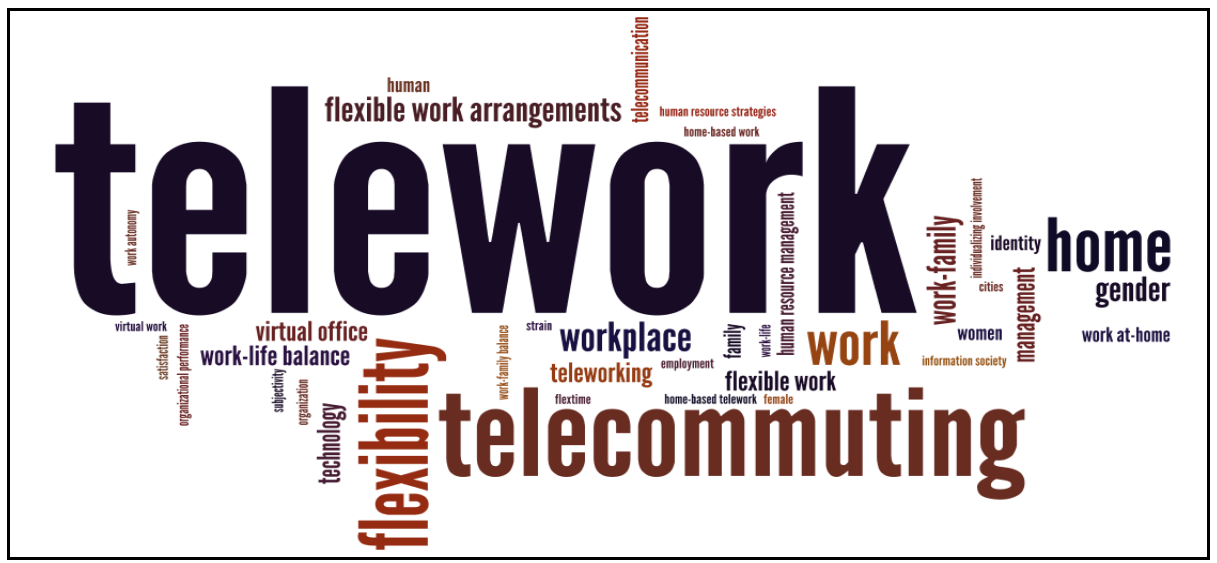

Fuente: Desarrollada por el autor.

Los autores utilizaron un total de 421 palabras clave en los 80 artículos. Las palabras que aparecieron con mayor frecuencia fueron telework (50), telecommuting (21), flexibility (13), home (10), work (9), workplace (6), work-family (5), flexible work arrangements (5) y gender (5). Las palabras más inseridas en las keyworks están relacionadas con las características de los empleados, tales como work-autonomy (2), childcare (1), choice (1), conflict (2), emotion (1), relationship (2), exhaustion (1), family (5), human resource (6), individual characteristics (2), satisfaction (5), life balance (5), motivators (1), psychology (3), responsiveness (1), support (1) y turnover (1). Palabras relacionadas con los aspectos internos de la empresa como company-performance, costcontrol, costs, flexible-firms, income, job performance e outcome fueron encontradas solo una vez. 
La Tabla 6 presenta los autores que han publicado más de dos artículos sobre el tema. También demostrada la cantidad de artículos publicados y de citaciones obtenidas (ICS y GSCS).

Tabla 6 - Cantidad de artículos publicados por autor y las citaciones recibidas

\begin{tabular}{llcccc}
\hline \multirow{2}{*}{$\mathbf{N}^{\circ}$} & \multicolumn{1}{c}{ Autores } & $\begin{array}{c}\mathbf{N}^{\circ} \text { de } \\
\text { artículos }\end{array}$ & $\%$ & \multicolumn{2}{c}{ Citaciones } \\
\cline { 5 - 6 } & & 04 & $5,0 \%$ & ICS & GSCS \\
\hline \hline 01 & Golden, T. D. & 04 & $5,0 \%$ & 00 & 135 \\
02 & de-Luis-Carnicer, P. & 04 & $5,0 \%$ & 00 & 83 \\
03 & Martinez-Sanchez, A. & 03 & $3,8 \%$ & 21 & 593 \\
04 & Hill, E. J. & 03 & $3,8 \%$ & 05 & 63 \\
05 & Johnson, L. C. & 03 & $3,8 \%$ & 00 & 81 \\
06 & Perez-Perez, M. & 03 & $3,8 \%$ & 00 & 46 \\
07 & Vela-Jimenez, M.J. & 02 & $2,5 \%$ & 04 & 59 \\
08 & Andrey, J. & 02 & $2,5 \%$ & 05 & 43 \\
09 & Greenhill, A. & 02 & $2,5 \%$ & 04 & 22 \\
10 & Maruyana, T. & 02 & $2,5 \%$ & 15 & 420 \\
11 & Miller, B. C. & 02 & $2,5 \%$ & 02 & 19 \\
12 & Moos, M. & 02 & $2,5 \%$ & 04 & 59 \\
13 & Shaw, S. M. & 02 & $2,5 \%$ & 02 & 19 \\
14 & Skaburskis, A. & 02 & $2,5 \%$ & 01 & 13 \\
15 & Stavrou, E. & 02 & $2,5 \%$ & 01 & 28 \\
16 & Taskin, L. & 02 & $2,5 \%$ & 00 & 00 \\
17 & Vink, P. & 02 & $2,5 \%$ & 05 & 43 \\
18 & Wilson, M. & 34 & $42,5 \%$ & 33 & 759 \\
19 & Otros autores (134) & $\mathbf{8 0}$ & $\mathbf{1 0 0} \%$ & $\mathbf{1 1 2}$ & $\mathbf{2 . 5 6 8}$ \\
\hline
\end{tabular}

Fuente: Desarrollada por el autor.

Entre los 152 investigadores encontrados en el análisis de los artículos, los autores Hill, E. J. y Miller, B. C. fueron los dos más citados, y juntos corresponden a el $40 \%$ del total de las 2.568 citaciones (GSCS). La Figura 4 fue generada con la ayuda del GraphMaker, disponible en el programa HistCite, y presenta la red de citaciones internas (ICS). Con el nombre resaltado, están los 17 artículos que fueron citados más de dos veces.

Figura 4 - Red de citaciones internas

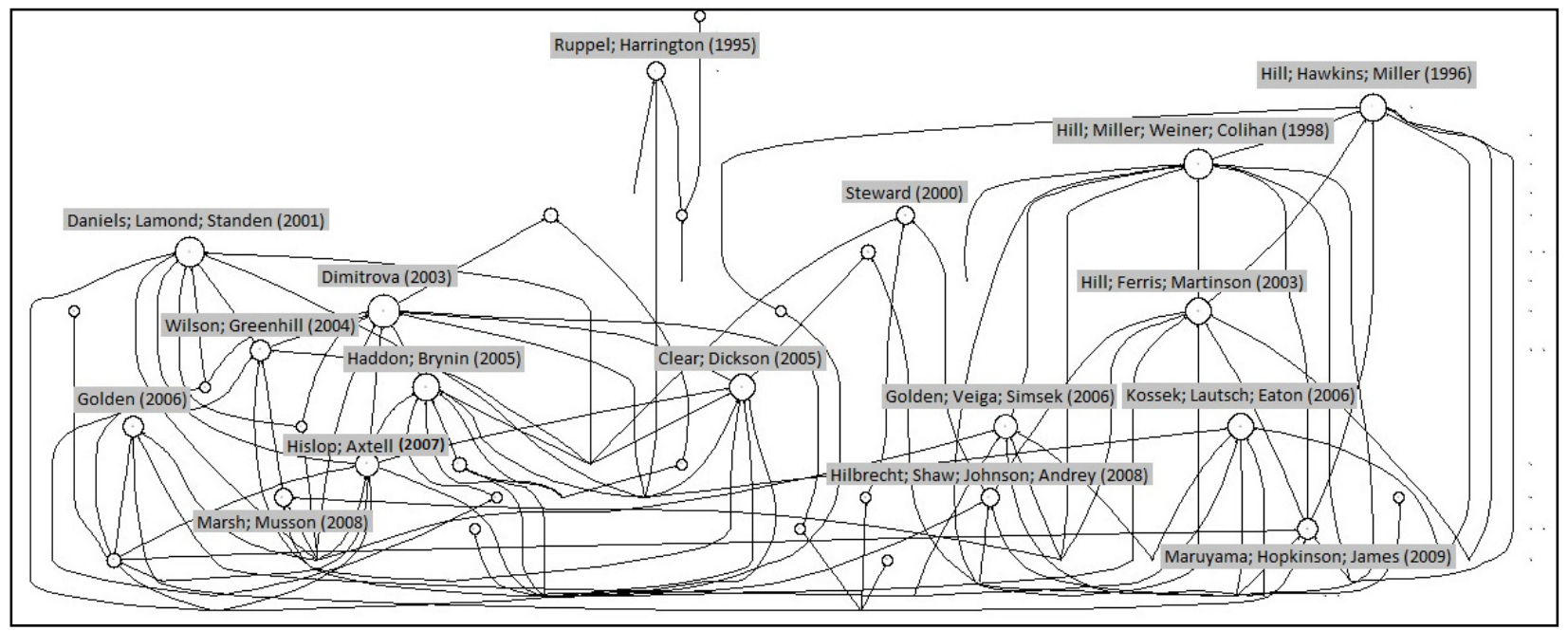

Fuente: Desarrollada por el autor. 
Finalmente, la Tabla 7 presenta los 15 artículos con el mayor índice de citaciones encontradas en el Google Académico (GSCS), el año en que fueron publicadas, el periódico y también los índices de citaciones (ICS y GSCS).

Tabla 7 - Cantidad de citaciones recibidas por los artículos

\begin{tabular}{|c|c|c|c|c|c|}
\hline \multirow{2}{*}{$\mathbf{N}^{\circ}$} & \multirow{2}{*}{ Autores de los artículos } & \multirow{2}{*}{ Año } & \multirow{2}{*}{ Periódico } & \multicolumn{2}{|c|}{ Citaciones } \\
\hline & & & & ICS & GSCS \\
\hline 01 & "Hill; Miller; Weiner; Colihan & 1998 & Personnel Psychology & 08 & 273 \\
\hline 02 & Kossek; Lautsch; Eaton & 2006 & Journal of Vocational Behavior & 06 & 182 \\
\hline 03 & Hill; Ferris; Martinson & 2003 & Journal of Vocational Behavior & 06 & 173 \\
\hline 04 & Sullivan; Lewis & 2001 & Gender Work and Organization & 00 & 150 \\
\hline 05 & Hill; Hawkins; Miller & 1996 & Family Relations & 07 & 147 \\
\hline 06 & Daniels; Lamond; Standen & 2001 & Journal of Management Studies & 08 & 121 \\
\hline 07 & Pratt & 1984 & Technological Forecasting and Social Change & 01 & 115 \\
\hline 08 & Hornung; Rousseau; Glaser & 2008 & Journal of Applied Psychology & 01 & 86 \\
\hline 09 & Golden; Veiga; Simsek & 2006 & Journal of Applied Psychology & 05 & 84 \\
\hline 10 & Shin; Sheng; Higa & 2000 & Journal of Org. Comp. and Elect. Commerce & 01 & 80 \\
\hline 11 & Dimitrova & 2003 & New Technology Work and Employment & 09 & 70 \\
\hline 12 & Mirchandani & 2000 & Qualitative Sociology & 02 & 67 \\
\hline 13 & Ruppel; Harrington & 1995 & Data Base for Advances Information Systems & 03 & 66 \\
\hline 14 & Steward & 2000 & Time \& Society & 03 & 65 \\
\hline 15 & Raghuram: London; Larsen & 2001 & International Journal of H.R. Management & 00 & 58 \\
\hline 16 & Otros artículos (65) & - & Otros periódicos (46) & 52 & 831 \\
\hline & & & TAL & 112 & 2.568 \\
\hline
\end{tabular}

Fuente: Desarrollada por el autor.

De acuerdo con la tabla anterior, la primera publicación entre las 15 más citadas fue hecha por Pratt (1984). El autor entrevistó a empleadores y trabajadores en busca de las principales ventajas percibidas por cada grupo encuestado. Para el empleador, las ventajas citadas fueron el acceso a una gran oferta de trabajo, un medio para la contratación a tiempo parcial, muy importante en sectores que tienen períodos de estacionalidad, y el aumento de productividad de los empleados. La ventaja principal señalada por los empleados fue la flexibilidad, pero también fueron encontradas ventajas como el aumento de la productividad y el ahorro del tiempo personal.

La referencia más citada fue la obra de Hill y otros, publicado en 1998 en el periódico Personnel Psychology, con ocho citaciones internas (ICS) y un total de 273 citaciones (GSCS). En este trabajo, los autores aplican una encuesta a los empleados de IBM, divididos en dos grupos (trabajadores tradicionales y teletrabajadores) y el análisis cualitativo reveló una percepción de mayor productividad, un aumento de la motivación, una mayor flexibilidad y una mayor carga de trabajo, así como una influencia negativa en el trabajo en equipo. Una parte de esta investigación había sido publicada por Hill, Hawkins y Miller (1996) y los resultados habían mostrado que los teletrabajadores percibieron una mayor flexibilidad laboral. Algunos también informaron que habían tenido éxito en la mejora de la relación con su familia debido a esta flexibilidad, mientras que otros reportaron que había una disputa familiar, ya que los límites entre la vida personal y profesional no estaban muy bien definidos.

Hill continuó estudiando los efectos del teletrabajo y en 2003 publicó un estudio junto con Ferris y Martinson, ahora evaluando tres formas de 
trabajo, la tradicional, la oficina virtual y la oficina en casa. Los resultados indicaron que la oficina virtual posee los aspectos más positivos para el trabajo. Cuanto a la conciliación entre la vida personal y profesional, el trabajo tradicional se presentó como lo que tiene el efecto más negativo, seguido de la oficina virtual y de la oficina en casa, que fue la que logró los mejores resultados. Shin, Sheng y Higa (2000) llevaron a cabo una revisión de la literatura sobre el teletrabajo y detectaron varios problemas, como la falta de una metodología sólida de investigación y un enfoque desproporcional en relación con los problemas personales de los teletrabajadores.

Un estudio de los teletrabajadores y sus compañeros de hogar fue realizado por Sullivan y Lewis (2001) y los resultados indican que, en términos de género, el teletrabajo puede mejorar al mismo tiempo el equilibrio entre la vida personal y profesional, así como dar continuidad al trabajo tradicional y a los roles familiares. Daniels, Lamond y Standen (2001) trataron de avanzar en los conceptos y desarrollaron un modelo explicativo, como un medio de integrar la literatura sobre la incidencia del teletrabajo y proporcionar una base teórica consistente para la comprensión del crecimiento de este tipo de trabajo en diferentes organizaciones.

El estudio de Kossek, Lautsch y Eaton (2006) levantó la percepción de los teletrabajadores de dos grandes compañías del periódico Fortune 500 y los resultados mostraron que los trabajadores que perciben un mayor control sobre el trabajo tenían un menor índice de rotación. Sin embargo, ellos relataron con mayor frecuencia que tenían conflictos entre la familia y el trabajo y también presentaron mayor índice de depresión. El estudio también encontró que los niveles más bajos de depresión fueron reportados por las mujeres que tenían hijos, lo que puede señalar un grupo donde el teletrabajo suele generar mejores resultados.

Golden, Veiga y Simsek (2006) estudiaron como el impacto del teletrabajo interfiere en las relaciones con la familia (work-to-family) y cómo las relaciones familiares interfieren en el trabajo (family-to-work) y encontraron que la autonomía de trabajar modera positivamente el workto-family y el tamaño de la familia modera negativamente el conflicto family-to-work. Estos resultados apuntan a variables que pueden afectar a la percepción de los individuos $y$, dependiendo del estudio que se propone, deberían ser consideradas como variables de control.

Finalmente, el trabajo más reciente (HORNUNG; ROUSSEAU; GLASER, 2008) trató de comprender los acuerdos de trabajo realizados por una agencia del gobierno alemán. Los acuerdos de horario flexible de trabajo mostraron una relación negativa a los conflictos familiares, mientras que los acuerdos de oportunidades de desarrollo presentaron relación positiva, ya que los empleados clasificados en esta modalidad se mostraron más comprometidos con la organización.

El análisis cualitativo de los artículos más citados, entre los seleccionados para este estudio, mostró que se ha invertido mucho esfuerzo en la búsqueda de la comprensión de los aspectos relacionados con la conciliación de la vida personal y profesional, sobre todo a través 
del prisma de los efectos psicológicos de las expectativas, del compromiso, del estrés y de la relación con la familia. Así como lo relatado por Shin Sheng y Higa (2000), fue posible percibir una mayor atención a las cuestiones relacionadas con los teletrabajadores, permaneciendo todavía un bajo interés en las investigaciones relacionadas con los efectos internos de la empresa, especialmente en relación a el impacto que el teletrabajo puede generar a la estructura organizacional de costes, a la productividad y al desempeño financiero.

\section{Conclusiones y recomendaciones de trabajos futuros}

Este artículo tuvo como objetivo mapear y analizar producciones científicas que exploran la flexibilidad y el teletrabajo, con el apoyo de una revisión sistemática de la literatura. El análisis de datos, la síntesis y la interpretación de los resultados posibilitaron presentar una visión general de cómo está la producción académica en relación al tema abordado.

A partir de las búsquedas realizadas fue posible ver que este tema ha ganado importancia, ya que lo años de mayor incidencia de publicaciones fueron 2012, 2010 y 2008. Este aumento puede estar asociado a la crisis internacional que empezó con la burbuja inmobiliaria americana, que salió a la luz en 2008 y que llevó inestabilidad e imprevisibilidad a prácticamente todos los mercados internacionales, que son dos factores fuertemente asociados al uso de la flexibilidad. Como contribución, los resultados de este estudio muestran la preferencia pelo idioma inglés en las publicaciones sobre el tema, así como el predominio de instituciones norte americanas (EE.UU. y Canadá) y del Reino Unido, tanto en el número de artículos como en la cantidad de citaciones.

Además, los resultados encontraron pocos estudios realizados en cooperación internacional. En este contexto, se entiende que todavía hay mucho que evolucionar, ya que el intercambio de experiencias entre investigadores de diferentes países es esencial para el fortalecimiento de la temática y para el avance de la ciencia. Este trabajo también identificó los artículos y periódico más citados, lo que permitió agruparlos por similitud, facilitando la comprensión de las principales contribuciones de cada uno en el desarrollo del tema, percibiendo que todavía hay espacio para investigaciones que avancen en la dirección del impacto del teletrabajo dentro en la empresa.

Por fin, este trabajo posibilita a otros investigadores continuar investigando con el uso de los mismos criterios utilizados o explorar otras bases de datos que no fueron contempladas. También es posible ampliar el análisis cualitativo, ya que para este trabajo sólo fueron considerados los 15 artículos más citados.

\section{Referencias}

ARAÚjO, C. A. Bibliometria: evolução história e questões atuais. Em Questão: Revista de Biblioteconomia \& Comunicação, v. 12, n. 1, p. 1132, 2006. 
BRADFORD, S. C. Sources of information on specific subject. Engineering, v. 26, p. $85-86,1934$.

CLEAR, F; DICKSON, K. Teleworking practice in small and medium-sized firms: management style and worker autonomy. New Technology Work and Employment, v. 20, n. 3, p. 218-233, 2005.

DANIELS, K.; LAMOND, D.; STANDEN, P. Teleworking, framework for organizational research. Journal of Management Studies, v. 38, p. 11511185. 2001.

DAVENPORT, T.; PEARLSON, K. Two cheers for the virtual office. Sloan Management Review, v. 39, n. 4, p. 51-65, 1998.

de LUIS, P. et al. Medioambiente y género: el caso de Aragón. En: JORNADAS DE ECONOMÍA CRÍTICA, 10., mar. 2006. Anales... Barcelona, 2006. p. 1-32.

DIMITROVA, D. Controlling teleworkers: supervision and flexibility revisited. New Technology Work and Employment, v. 18, n. 3, p. 181-195, 2003.

GOLDEN, T. D. Avoiding depletion in virtual work: telework and the intervening impact of work exhaustion on commitment and turnover intentions. Journal of Vocational Behavior, v. 69, n. 1, p. 176-187, 2006.

GOLDEN, T. D.; VEIGA, J. F.; SIMSEK, Z. Telecommuting's differential impact on work-family conflict: Is there no place like home? Journal of Applied Psychology, v. 91, n. 6, p. 1340-1350, 2006.

HADDON, L; BRYNIN, M. The character of telework and the characteristics of teleworkers. New Technology Work and Employment, v. 20, n. 1, p. 3446. 2005

HART, A. Anticipations, business planning and the cycle. Quarterly Journal of Economics, p. 272-293, 1937.

HILBRECHT, M.; SHAW, S. M.; JOHNSON, L. C.; ANDREY, J. I'm home for the kids: contradictory Implications for work-life balance of teleworking mothers. Gender Work and Organization, v. 15, n. 5, p. 454-476, 2008.

HILL, E. J.; FERRIS, M.; MARTINSON, V. Does it matter where you work? A comparison of how three work venues (traditional office, virtual office, and home office) influence aspects of work and personal/family life. Journal of Vocational Behaviour, v. 63, n. 2, p. 220-241, 2003.

HILL, E. J.; HAWKINS, A. J.; MILLER, B. C. Work and family in the virtual office - Perceived influences of mobile telework. Family Relations, v. 45, n. 3, p. 293-301, 1996.

HILL, E. J. et al. Influence of the virtual office on aspects of work and work/life balance, Personnel Psychology, v. 51, n. 3, p. 667-683, 1998.

HISLOP, D.; AXTELL, C. The neglect of spatial mobility in contemporary studies of work: the case of telework. New Technology Work and Employment, v. 22, n. 1, p. 34-51, 2007. 
HORNUNG, S.; ROUSSEAU, D. M.; GLASER, J. Creating flexible work arrangements through idiosyncratic deals. Journal of Applied Psychology, v. 93, n. 3, p. 655-664, 2008

JACKSON, N. Unit One: background to systematic reviews. Handbook: systematic reviews of health promotion and public health interventions. 2004. Disponível em: <http://ph.cochrane.org/sites/ph.cochrane.org/files/uploads/Unit_One.pdf >. Acesso em: 05 jun. 2013.

KOSSEK, E. E.; LAUTSCH, B. A.; EATON, S. C. Telecommuting, control, and boundary management: Correlates of policy use and practice, job control, and work-family effectiveness. Journal of Vocational Behavior, v. 68, n. 2, p. 347-367, 2006.

MARSH, K.; MUSSON, G. Men at work and at home: Managing emotion in telework. Gender Work and Organization, v. 15, n. 1, p. 31-48, 2008.

MARUYAMA, T.; HOPKINSON, P. G.; JAMES, P. W. A multivariate analysis of work-life balance outcomes from a large-scale telework programme. New Technology Work and Employment, v. 24, n. 1, p. 76-88, 2009.

MIRCHANDANI, $\mathrm{K}$. The best of both worlds and cutting my own throab: contradictory images of home-based work. Qualitative Sociology, v. 23, n. 2, p. 159-182, 2000.

NATIONAL HEALTH SERVICE (NHS). CENTRE FOR REVIEWS AND DISSEMINATION. Undertaking systematic reviews of research on effectiveness. New York: University of New York, 2001.

PRATT, J. H. Home teleworking: a study of its pioneers. Technological forecasting and social change, v. 25, n. 1, p. 1-14, 1984.

RUPPEL, C.; HARRINGTON, S. Telework: na innovation where nobody is getting on the band-wagon. Database, v. 26, n. 2-3, p. 87-104, 1995.

SANCHEZ, A. M. et al. Teletrabajo, flexibilidad de recursos humanos y resultados de la empresa. Management, v. 12, n. 1, p. 52-79, 2009.

SANCHEZ, R. Strategic flexibility in product competition. Strategic Management Journal, v. 16, special issue, p. 135-159, 1995.

SHIN, B.; SHENG, O.; HIGA, K. Telework: existing research and future directions. Journal of Organizational Computing and Electronic Commerce, v. 10, n. 2 , p. 85-101. 2000.

STEWARD, B. Changing times: the meaning, measurement and use of time in teleworking. Time and Society, v. 9, n. 1, p. 57-74, 2000.

STIGLER, G. Production and distribution in the short run. Journal of Political Economy, v. 47, n. 3, p. 305-327, 1939.

SULLIVAN, C.; LEWIS, S. Home-based telework, gender, and the synchronization of work and family: perspective of teleworkers and their co-residents. Gender, work and Organization, v. 8, n. 2, p. 123-145, 2001. 
RAGHURAM, S.; LONDON, M.; LARSEN, H. H. Flexible employment practices in Europe: country versus culture. International Journal of Human Resource Management, v. 12, n. 5, p. 738-753, 2001.

UPTON, D. The management of manufacturing flexibility. California Management Review, v. 36, n. 2, p. 72-89, 1994.

WILSON, M.; GREENHILL, A. Gender and teleworking identities in the risk society: A research agenda. New Technology, Work and Employment, v. 19, n. 3, p. 207-221, 2004.

WRIGHT, P. M.; SNELL, S. A. Toward a unifying framework for exploring fit and flexibility in strategic human resource management. Academy of Management Review, v. 23, p. 756-772, 1998. 